\title{
Target detection with a liquid-crystal-based passive Stokes polarimeter
}

\author{
François Goudail, Patrick Terrier, Yoshitate Takakura, Laurent Bigué, Frédéric Galland, \\ and Vincent DeVlaminck
}

\begin{abstract}
We present an imaging system that measures the polarimetric state of the light coming from each point of a scene. This system, which determines the four components of the Stokes vector at each spatial location, is based on a liquid-crystal polarization modulator, which makes it possible to acquire fourdimensional Stokes parameter images at a standard video rate. We show that using such polarimetric images instead of simple intensity images can improve target detection and segmentation performance. (C) 2004 Optical Society of America
\end{abstract}

OCIS codes: $\quad 120.5410,260.5430,100.0100,100.5010,230.3720$.

\section{Introduction}

The polarization state of light contains important information about a scene that is complementary to information provided by the light's intensity and color. Forming an image of the polarization properties of the light reflected or emitted by a scene is thus useful in such applications as scene analysis, robotics, and automatic target recognition. ${ }^{1-3}$ Such an image can reveal contrasts between two zones of the scene that have the same intensity reflectivity (so that no contrast appears in the intensity image) but different polarimetric properties, which can improve the detectability of small, low-contrast objects in images. ${ }^{4-6}$

Polarimetric imaging systems can be classified into two categories: passive and active. An active system uses an artificial light source to illuminate the scene. In a passive system the image is formed from a reflection of the ambient light ${ }^{7,8}$ or from the radia-

F. Goudail (francois.goudail@fresnel.fr) and F. Galland are with the Fresnel Institute, Unité Mixte de Recherche au Centre National de la Recherche Scientifique (UMR CNRS 6133), Marseille, France; P. Terrier and V. DeVlaminck are with the Laboratoire Interaction, Image et Ingénierie de la Décision (FRE CNRS 2497), Lille, France; Y. Takakura is with the Laboratoire des Sciences de l'Image, de l'Information et de la Télédétection (UMR CNRS 7005), Strasbourg, France; L. Bigué is with the Laboratoire de modélisation, Intelligence, Processus, et Systèms (MIPS 2332), Mulhouse, France.

Received 14 April 2003; revised manuscript received 11 September 2003; accepted 8 October 2003.

0003-6935/04/020274-09\$15.00/0

(C) 2004 Optical Society of America tion emitted in an IR system. ${ }^{6,9,10}$ A polarimetric camera measures the polarization state of the light incident upon the camera, and its usual output is a set of Stokes parameter images. By analyzing the direction of polarization and the degree of partial polarization of the reflected light, one can obtain important information about the presence and the orientation of occluding edges ${ }^{3,7}$ or about the surface orientation of the observed objects.3,11-13 Another important application of polarimetric imagery is in the determination from their polarimetric properties of the nature of the materials that are present in a scene. ${ }^{14}$ Polarimetric imagery has been found useful in detecting rust during inspection of ships' hulls ${ }^{13}$ and in discriminating between dielectric and metallic areas on circuit boards, ${ }^{3,12}$ which can be difficult with intensity or even color images.

We describe in this paper a passive imaging system that measures the Stokes vector at each spatial location in a scene. It uses a variable retardance scheme, ${ }^{15}$ and the polarimetric modulation is performed by two liquid-crystal variable retarders (LCVRs). Because of the rapid action of these devices, Stokes parameter images can be acquired and formed at a standard video rate. The LCVRs are key components of the system, and we characterize their performance in terms of precision of modulation and image quality with a high-precision Mueller imager. Finally, we address the important problem of efficiently extracting information from these vectorial images. For that purpose we consider statisticsbased detectors and a state-of-the-art segmentation algorithm based on statistically active contours and the principle of minimum description length 


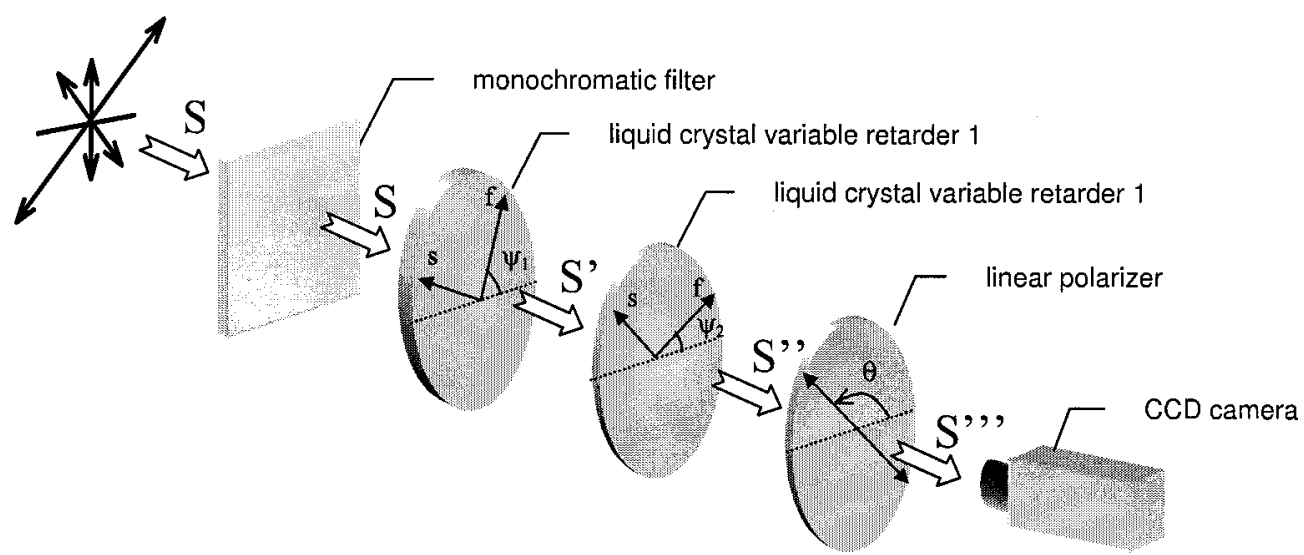

Fig. 1. Schematic of the proposed Stokes imaging polarimeter.

(MDL). ${ }^{16}$ We demonstrate the efficiency of these algorithms for the detection of small objects in Stokes images and for the characterization of their polarimetric properties.

In Section 2 we describe the Stokes imaging system and characterize the polarimetric response of the LCVRs used in the system to assess the precision of their measurements. In Section 3 we address the issue of designing efficient processing techniques for vectorial polarimetric images. After characterizing the statistical properties of the noise that affects the images and describing the operation of the imageprocessing algorithms that we shall consider, we illustrate the performance of the algorithms with several images acquired by the Stokes imaging system.

\section{Stokes Imaging System}

The measurement device is based on the use of two Meadowlark Optics LRC-300 LCVRs. Each one of these optical components makes it possible to modify the polarization state of the incident light wave without requiring mechanical actions on the device (rotations). The Stokes parameters of the incident light, $\mathbf{S}=\left[S_{0}, S_{1}, S_{2}, S_{3}\right]^{T}$ ( $T$ denotes transposition), can thus be estimated precisely and rapidly. In the present case the variable parameter of the device is not an angular position but a pair of retardances $\left(\delta_{1}\right.$, $\delta_{2}$ ). These retardances are adjusted by the amplitude of a rectangular alternative voltage that is applied to the LCVR through an intermediately located command interface board. First we describe the operation of the proposed Stokes polarimeter, and then we characterize the polarimetric response of the LCVR.

\section{A. Operation of the Stokes Imaging Polarimeter}

A schematic of the device is illustrated in Fig. 1. A light wave passes successively through a monochromatic filter centered on wavelength $\lambda_{0}=520 \mathrm{~nm}$ (middle of the visible spectrum), two variable retarders, and a linear polarizer. The resultant light intensity is then measured by a CCD camera. The influence of each of these optical components on the state of polarization of the light wave is modeled by a Stokes-Mueller formalism. Stokes vector $\mathbf{S}^{\text {out }}$ of the light wave at the polarizer output (denoted $\mathbf{S}^{\prime \prime \prime}$ in Fig. 1 ) is thus related to input Stokes vector $\mathbf{S}^{\text {in }}$ (denoted $\mathbf{S}$ in Fig. 1) by the following matrix multiplication:

$$
\mathbf{S}^{\text {out }}=M_{\mathrm{POL}} M_{R}\left(\delta_{1}\right) M_{R}\left(\delta_{2}\right) \mathbf{S}^{\text {in }}=M_{\text {global }} \mathbf{S}^{\text {in }}
$$

where $M_{R}(\delta)$ is the Mueller matrix of a pure retarder with retardance $\delta$ and $M_{\mathrm{POL}}$ is the Mueller matrix of a linear polarizer. ${ }^{15}$ For a given pair of retardances $\left(\delta_{1}, \delta_{2}\right)$, light intensity $I_{i}$ measured by the CCD camera corresponds to Stokes parameter $S_{0}^{\text {out }}$. It is thus a linear function of the input Stokes parameters $\mathbf{S}^{\text {in }}$ of the observed light:

$$
\begin{aligned}
I= & S_{0}^{\text {out }} \\
= & A\left(\delta_{1}, \delta_{2}\right) S_{0}^{\text {in }}+B\left(\delta_{1}, \delta_{2}\right) S_{1}^{\text {in }}+C\left(\delta_{1}, \delta_{2}\right) S_{2}^{\text {in }} \\
& +D\left(\delta_{1}, \delta_{2}\right) S_{3}^{\text {in }},
\end{aligned}
$$

where the parameters $[A, B, C, D]$ correspond to the first line of $M_{\text {global }}$. As a consequence, for each pixel of the image of the observed scene, one can estimate the four parameters of Stokes vector $\mathbf{S}$ by carrying out $N$ acquisitions $\mathbf{I}=\left\{I_{i}, i \in[1, N]\right\}$ for $N$ combinations of delays $\left(\delta_{1}, \delta_{2}\right)$ :

$$
\mathbf{I}=M_{\mathrm{LIGHT}} \mathbf{S} \leftrightarrow\left(\begin{array}{c}
I_{1} \\
I_{2} \\
\vdots \\
\dot{I}_{N}
\end{array}\right)=\left[\begin{array}{cccc}
A_{1} & B_{1} & C_{1} & D_{1} \\
A_{2} & B_{2} & C_{2} & D_{2} \\
\vdots & \vdots & \vdots & \vdots \\
\dot{A_{N}} & B_{N} & \dot{C}_{N} & D_{N}
\end{array}\right]\left(\begin{array}{c}
S_{1} \\
S_{2} \\
S_{3} \\
S_{4}
\end{array}\right),
$$

where matrix $M_{\text {LIGHT }}$ is a function of the $N$ couples of delays (one couple per line) and of the positioning of the optical components. For expression (3) to be invertible, $N$ must be equal at least to 4 . However, in practice, better results are obtained with larger numbers of measurements. When $N>4$, one can obtain 
the Stokes parameters by minimizing function $F$, given by

$$
F=\sum_{i=1}^{N}\left[I_{i}-\left[A_{i}, B_{i}, C_{i}, D_{i}\right]\left(\begin{array}{c}
S_{1} \\
S_{2} \\
S_{3} \\
S_{4}
\end{array}\right)\right]^{2} .
$$

The positioning of each optical component $\left(\psi_{1}, \psi_{2}\right.$, and $\theta$ in Fig. 1) is chosen to produce parameters $A_{i}, B_{i}, C_{i}$, and $D_{i}$ that correspond to sufficient conditioning of matrix $M_{\text {LIGHT }}$ that expression (3) can be safely inverted. ${ }^{17}$

The maximum acquisition speed of 25 images/s (standard video rate) implies that Stokes vector $\mathbf{S}$, for all the pixels of the imaged scene, is obtained in a minimum time of $160 \mathrm{~ms}$. However, in practice, obtaining a more robust estimate of the Stokes parameters often requires a sequence of at least $N=8$ acquisitions. A $320-\mathrm{ms}$ period is thus necessary to produce an overdetermination of the system of equations.

It is important to note that, in the experimental setup considered here, the light illuminating the scene may be supposed to be diffuse or nonpolarized. The observed polarimetric effect comes from the capacity of the various materials in the scene to polarize light. One of the physical phenomena that can lead to this effect is Fresnel reflection at a dielectric interface. Indeed, during this process the components parallel and orthogonal to the plane of incidence undergo different coefficients of reflection, which can create a partial polarization of the light. ${ }^{18}$

\section{B. Characterization of the Polarimetric Response of the LCVRs}

The LCVRs are key components of the proposed Stokes imaging system, and they determine the accuracy of its measurements. It is thus important to characterize their properties. The LRC-300 LCVRs used in the measurement system are constructed by use of optically flat fused-silica windows and nematic liquid-crystal materials. These true zero-order retarders are designed for maximum transmission from 400 to $700 \mathrm{~nm}$. With no voltage applied, the liquidcrystal molecules lie parallel to the glass substrates, and maximum retardation is achieved. When voltage is applied, liquid-crystal molecules begin to tip in a direction perpendicular to the fused-silica windows. As voltage increases, the molecules tip further, causing a reduction in the effective birefringence and, hence, retardance. The response time for the LCVR to switch from one-half to zero wave (low to high voltage) is $\sim 5 \mathrm{~ms}$; it is $20 \mathrm{~ms}$ for switching from zero to one-half wave (high to low voltage).

We are interested in a full characterization of polarization controllers, as suggested by Drewes and Chipman, ${ }^{19}$ and in studying the spatial response of the LCVR by taking an imaging approach. For this purpose we used the imaging Mueller polarimeter situated at the Laboratoire des Sciences de l'Image, de l'Information et de la Télédétection, Strasbourg. ${ }^{20}$
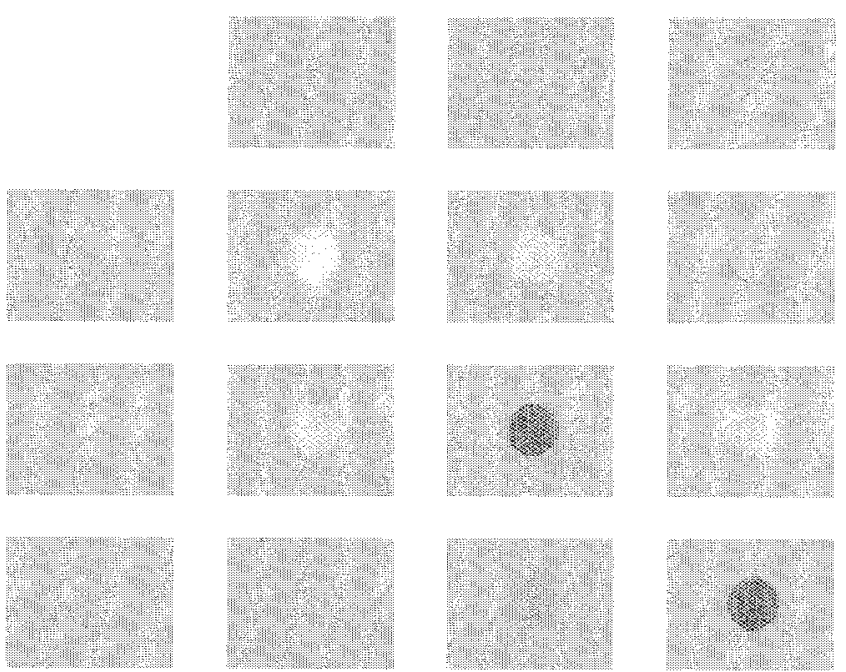

Fig. 2. Mueller image of the LCVR. Each subimage represents the image of a coefficient of the Mueller matrix. The gray scale is such that negative values appear in dark colors, zero in medium gray, and positive in lighter gray. All images are normalized with respect to the positive part of image $M_{00}$, which thus appears completely white here.

This system can accurately measure the Mueller matrix at each point of a scene. The advantage of such device compared with a standard nonimaging polarimeter is that it makes possible the estimation of the spatial homogeneity of the polarimetric response. In fact, for liquid-crystal modulators the lack of spatial homogeneity is often the main problem. However, we tested regions at the center and on the edges of the device, and the device's response proved quite homogeneous. Figure 2 gives examples of Mueller images obtained for a 2-V input voltage, corresponding to an approximate half-wave operation, when a binary pattern is placed in the input beam. It can be seen that the Mueller response corresponds to that of a pure retarder. ${ }^{15}$ Up to the accuracy of the imaging setup, the diffusion or depolarization effects that characterize some liquid-crystal modulators have not been observed. Moreover, the contrast of the binary pattern is preserved, which indicates good spatial resolution of the device.

\section{Target Detection and Characterization in Polarimetric Images}

In this section we show some examples of applications in which the Stokes parameter image, compared to simple intensity imaging, can improve information extraction from a scene. One of the advantages of precise Stokes imaging is that it reveals slight variations in the polarimetric properties of the objects that are present in a scene. Because the contrasts are weak, it is necessary to take into account the nature of the noise that is present in the image to be able to perform efficient information extraction. Algorithms based on statistical decision and estimation theory are good candidates for addressing this problem. 


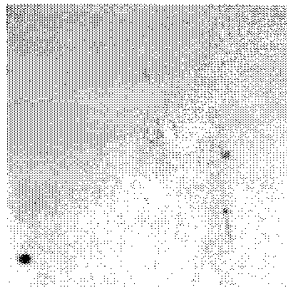

$S_{0}$

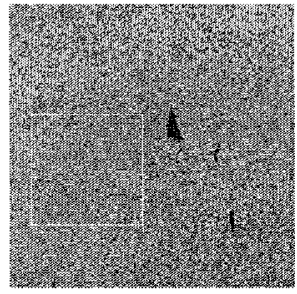

$S_{1}$

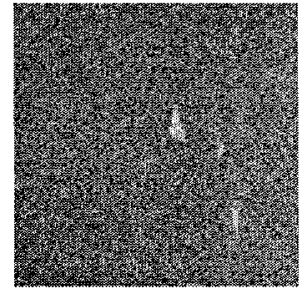

$S_{2}$

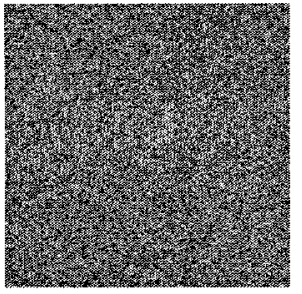

$S_{3}$

Fig. 3. Stokes parameter image of three small pieces of transparent cellophane tape on a cardboard background.

In Subsection 3.A we determine the nature of the noise that is present in the images of interest to be able to design detection and segmentation algorithms. The characteristics of such algorithms are described in Subsection 3.B, and they are applied in Subsections 3.C and 3.D to images acquired with the proposed Stokes imager.

\section{A. Characterization of Noise}

Statistics-based detection and segmentation algorithms rely on a probabilistic model of the images. Let us characterize the noise that affects the images so we can determine an adequate image model from which processing algorithms will be derived. Figure 3 presents the four components of the Stokes parameter image acquired with the system described in Section 2 . Ten different pairs of retardances $(\delta 1, \delta 2)$ have been used, corresponding to every combination of $\delta_{1}=80^{\circ}, 240^{\circ}$ and $\delta_{2}=0^{\circ}, 80^{\circ}, 160^{\circ}, 240^{\circ}, 320^{\circ}$. For each of these ten pairs of retardances the CCD camera measures, for each pixel of the observed scene, light intensity $I_{i}$ used for minimization of function $F$ [Eq. (4)]. The positioning of the optical components is $\psi_{1}=20^{\circ}, \psi_{2}=45^{\circ}$, and $\theta=90^{\circ}$ (Fig. 1). These values lead to parameters $A_{i}, B_{i}, C_{i}$, and $D_{i}$, which allow for sufficient conditioning of matrix $M_{\text {LIGHT }}$ [expression (3)].

The scene represents three small objects on a uniform background. The background is made of cardboard, and the objects are small pieces of transparent cellophane tape. It is interesting to note that component $S_{0}$, which represents the intensity image, looks quite different from the three other channels.
The objects, which are transparent, do not appear clearly in this image, whereas some spots that are due to dust are quite apparent. In the three other channels, however, the objects of interest appear in clear contrast (positive or negative) to a rather uniform background. Indeed, these last three channels of the Stokes vector represent the polarimetric properties of the light. It can be seen that the light reflected from the cardboard background has homogeneous polarimetric properties, which are different from those of the light reflected from the objects of interest.

It can also be noted that the noise in channels $S_{1}$, $S_{2}$, and $S_{3}$ is quite important. This is so because the polarimetric contrast is weak and thus the dynamic range of the image is low. Let us now determine the statistical properties of this noise so we can design the processing algorithms. We consider the region outlined by a white rectangle in Fig. 3, channel $S_{1}$. We have represented in Fig. 4 histograms of this region for the three polarimetric channels $S_{1}, S_{2}$, and $S_{3}$. We have superimposed upon the histograms Gaussian curves with the same means and variances as the samples.

It can be seen that the noise is close to Gaussian. We thus consider an image model in which the noise is Gaussian. More precisely, to process the polarimetric data we consider a vectorial, three-channel image $\overline{\mathbf{S}}=\left(S_{1}, S_{2}, S_{3}\right)$ composed of the last three Stokes parameters. This vector will be the input to the detection and segmentation algorithms. Each of its channels will be assumed to be statistically independent and distributed with a Gaussian probabilitydensity function.
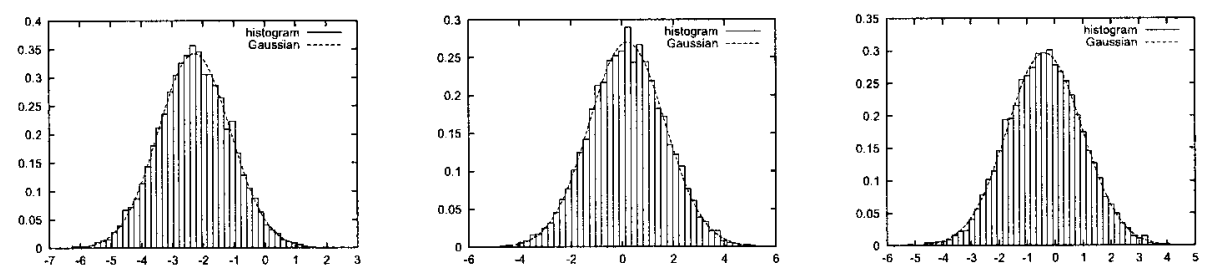

Fig. 4. Histograms of a homogeneous region in the three channels, $S_{1}, S_{2}$, and $S_{3}$. The region considered here is the area outlined in white in Fig. 3, channel $S_{1}$. A Gaussian with same mean and variance as the corresponding histogram is drawn (dotted curve) over each histogram. Mean $m$ and standard deviation $\sigma$ in the three channels are $m_{1}=-2.24$ and $\sigma_{1}=1.12$ in $S_{1} ; m_{2}=-0.20$ and $\sigma_{2}=1.46$ in $S_{2} ; m_{3}=-0.40$ and $\sigma_{3}=1.28$ in $S_{3}$. 


\section{B. Statistical Image Processing}

We describe in this subsection algorithms based on statistical decision and estimation theory that can solve problems of target detection and shape segmentation in the vectorial Stokes parameter images. We assume that the image- or the subimage- of interest is composed of two regions: region a, which corresponds to the object of interest, and region $b$, which corresponds to the background. The shape of the object, which corresponds to the borders between the two regions, is defined by a set of parameters $\mathbf{w}$. As explained above, the gray levels in each of these regions are assumed to be random vectors distributed with Gaussian probability-density functions. We describe, in what follows, detection and segmentation algorithms based on this image model.

\section{Target Detection Based on the Generalized Likelihood Ratio Test}

The problem that we address is detection of a target with shape $\mathbf{w}$ (which defines region a) in an image. For small targets, w can be defined as a square with the size of the expected targets. We define another shape, F, which contains $\mathbf{w}$, and we denote the complement of $\mathbf{w}$ in $\mathbf{F}: \quad \overline{\mathbf{w}}=\mathbf{F}-\mathbf{w}$. The number of pixels in shape $\mathbf{F}(\mathbf{w}, \overline{\mathbf{w}})$ is $N_{F}\left(N_{a}, N_{b}\right)$, with $N_{F}=$ $N_{a}+N_{b}$.

The image is scanned with mask $\mathbf{F}$ and, for each position $\boldsymbol{\tau}=(x, y)$ in the image, detection is made by use of a generalized likelihood ratio test (GLRT). ${ }^{21}$ Inasmuch as the Stokes channels are modeled with Gaussian probability-density functions with unknown means and variances, the GLRT consists in computing, for each position $\tau$, the following expres$\operatorname{sion}^{22}$ :

$$
\begin{aligned}
\mathscr{R}(\boldsymbol{\tau}, \mathbf{w})= & \sum_{k=1}^{3}\left\{-N_{a} \log \left(\left[\hat{\sigma}_{a}^{2}\right]_{k}\right)-N_{b} \log \left(\left[\hat{\sigma}_{b}^{2}\right]_{k}\right)\right. \\
& \left.+N_{F} \log \left(\left[\hat{\sigma}_{F}^{2}\right]_{k}\right)\right\},
\end{aligned}
$$

where $\left[\hat{\sigma}_{u}^{2}\right]_{k}$ is the variance empirically estimated in regions a, $\mathrm{b}$, and $\mathrm{F}$ in channel $S_{k}$. We then compare $\mathscr{R}(\boldsymbol{\tau}, \mathbf{w})$ with a threshold to determine whether a target is present at position $\tau$.

In practice, the sizes of the searched-for objects may not be known. The size can then be considered as a nuisance parameter that can be estimated in the maximum-likelihood sense. More precisely, let us denote by $\left\{\mathbf{w}_{k}, k \in[1, K]\right\}$ the $K$ possible shapes. The detection criterion that will be used has the following expression $^{23}$ :

$$
\mathscr{R}(\boldsymbol{\tau})=\max _{k}\left[R\left(\boldsymbol{\tau}, \mathbf{w}_{k}\right)\right] .
$$

It consists of choosing the mask $\mathbf{w}_{k}$ that leads to the highest value of the GLRT.

\section{Target Segmentation}

Let us now consider segmentation of a single object in an image. To solve this problem, we use a method based on polygonal active contours and on the MDL principle. ${ }^{16}$ This method consists in determining the polygon with the minimal number of nodes that best approximates the shape of the object, in the sense of the MDL criterion. ${ }^{16,24,25}$ We deform the polygon and determine its number of nodes by optimizing a mathematical criterion that depends on the number and the position of the nodes of the polygon. w denotes the shape of the object, that is, the coordinates of the nodes of polygonal shape, $k$ is the number of nodes of the polygon, and $N$ is the total number of pixels in the image. In the case where the noise in each channel is Gaussian, the expression of the mathematical criteria is

$$
\begin{aligned}
J(\mathbf{w}, k)= & \sum_{k=1}^{3}\left(N_{a}(\mathbf{w}) \log \left\{\left[\hat{\sigma}_{a}^{2}\right]_{k}(\mathbf{w})\right\}\right. \\
& \left.+N_{b}(\mathbf{w}) \log \left\{\left[\hat{\boldsymbol{\sigma}}_{b}^{2}\right]_{k}(\mathbf{w})\right\}\right)+k \log N,
\end{aligned}
$$

where $N_{a}(\mathbf{w})$ and $N_{b}(\mathbf{w})$ are the number of pixels on the target and on the background, respectively, which depend on the shape of the object $\mathbf{w}$. This criterion corresponds to an approximation of the average code length necessary for encoding the image data. ${ }^{16}$

In practice, this criterion is optimized in two steps. ${ }^{16}$ In the first step we segment the object by optimizing $J(\mathbf{w}, k)$ with respect to $\mathbf{w}$ for an increasing number of nodes $k$ to obtain a precise estimation of the object's shape. The second step consists in removing the less-useful nodes (i.e., decreasing $k$ ) one by one until the criterion $J(\mathbf{w}, k)$ is minimized. It is worth noting that this segmentation algorithm possesses no free parameter to be adjusted by the user. Examples of application of this method are shown in Subsection 3.C.

\section{Determination of the Shapes of Large Objects}

Let us consider first the segmentation of complex shapes in Stokes images. Figure 5 presents images of two shapes made from transparent cellophane tape on a cardboard background. The physical configuration that we used to obtain these images is the same as that used for Fig. 3. The shapes are barely visible on intensity images $S_{0}$, which are also perturbed by dust. In channels $S_{1}-S_{3}$, however, the shapes appear in clear contrast (positive of a negative) to the background. As we said above, we thus work on the vectorial three-channel image composed of Stokes components $S_{1}-S_{3}$, to which we apply the multichannel snake defined in Eq. (7). We present in Fig. 6 the result of the segmentation of these objects. The two leftmost figures represent the initial shape, which is a rectangle. The two central figures represent the polygonal contour estimated after the first step of the segmentation algorithm, which consists in progressively adding nodes to the contour to obtain a precise segmentation. It can be seen that the global shapes are correctly segmented but that there are many spurious nodes. Finally, the rightmost two figures represent the result of the second step, in which the less useful nodes are pruned. One can see that the objects are accurately segmented with a limited number of nodes. These accurate and 

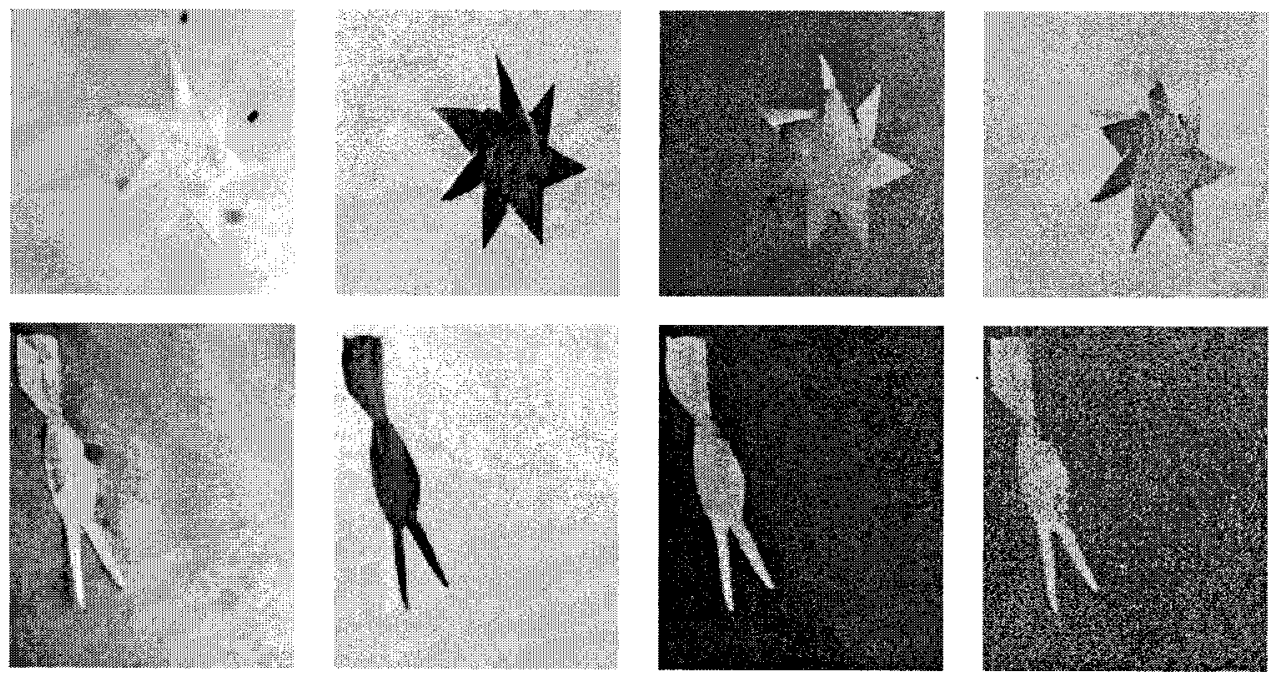

$S_{0}$

$S_{1}$

$S_{2}$

$S_{3}$

Fig. 5. Stokes parameter images of two objects.

parsimonious shape estimates could be used in shape recognition systems, for example.

D. Detection and Characterization of Regions of Interest

Let us now consider the detection of the three small objects in the Stokes parameter image of Fig. 3. As we said above, we are working on a vectorial threechannel image composed of the Stokes components $S_{1}, S_{2}$, and $S_{3}$. We apply the multiscale GLRT defined in Eq. (6), where mask $\mathrm{F}$ is a rectangle of
$39 \times 13$ pixels and four masks $\mathbf{w}_{k}, \mathbf{k} \in[1,4]$ are considered: They are rectangles of sizes $5 \times 3$, $11 \times 5,21 \times 7$, and $31 \times 9$. The GLRTs adapted to these masks are applied to the image, and the results are fused according to Eq. (6). The likelihood ratio plane $\mathscr{R}(\boldsymbol{\tau})$ is represented in Fig. 7, together with vertical and horizontal cross sections. The positions of the three objects of interest appear clearly in this image.

Once the regions of interest have been detected by
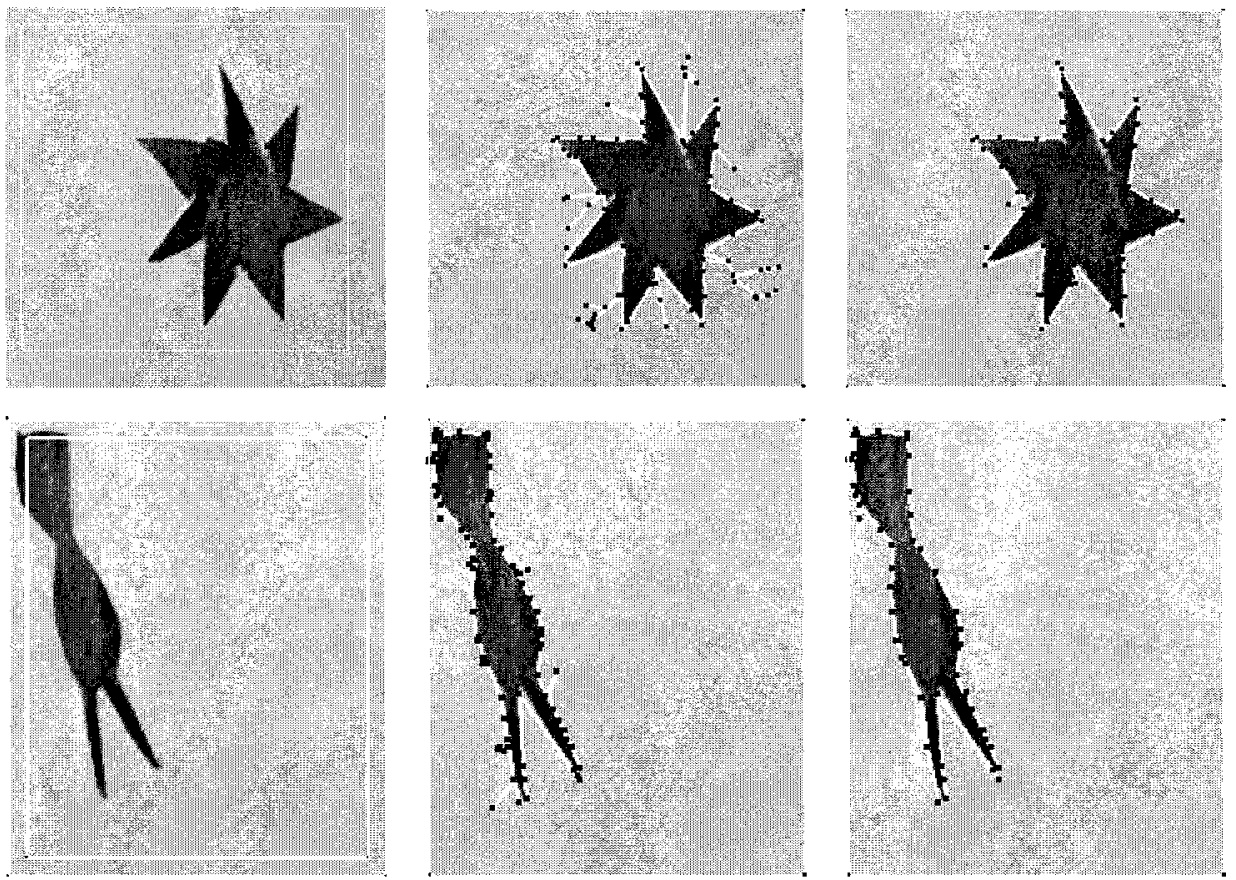

Fig. 6. Segmentation of the two objects in Fig. 5, in the reduced Stokes parameter images $\overline{\mathbf{S}}=\left\{S_{1}, S_{2}, S_{3}\right\}$. Left, initial shape (channel $S_{1}$ ); center, results of the first step of the segmentation process (segmentation with node adding); right, results of the second step of the segmentation process (node pruning). 

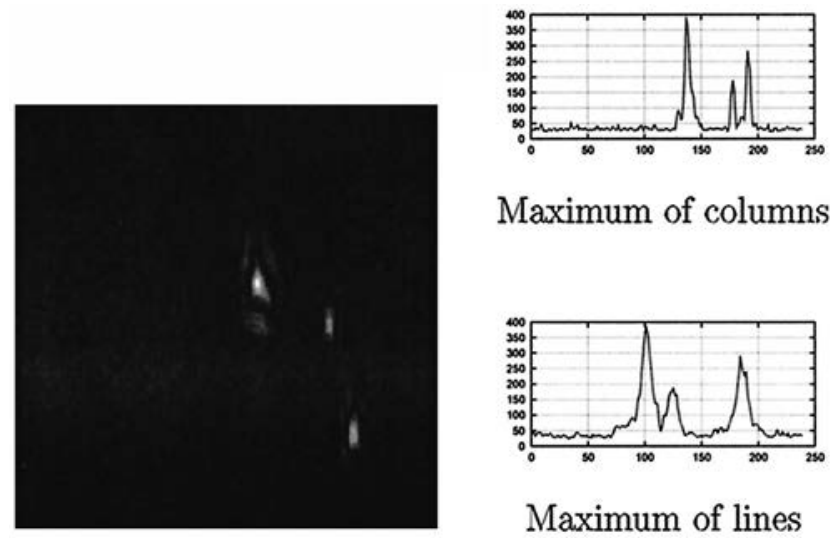

Maximum of columns

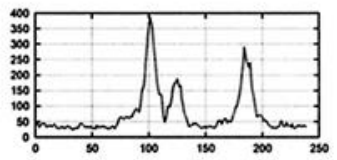

Maximum of lines

Fig. 7. Plane $\mathscr{R}(\boldsymbol{\tau})$ obtained with the multitarget GLRT on the image of Fig. 3; maximum of each column and of each line of this plane.

the GLRT, it can be useful to determine their shapes and to estimate their average polarization properties to characterize the nature of the objects. We see that the MDL-based snake algorithm can perform this operation efficiently.

Let us consider $50 \times 50$ pixel subimages centered on the detected regions of interest. The snake is initialized as a rectangular shape on the corner of the images, as shown in Fig. 8, upper row. The contour then evolves to segment the object, as can be seen from the bottom row of Fig. 8.

Once the shape of the object has been determined, it can be used to estimate some parameters, such as the average polarimetric properties. For example, we have estimated the average Stokes parameters within each object, and we have converted them into another representation of the polarimetric parame- ters: intensity $I$, degree of polarization $\mathscr{P}$, and polar angles $\varphi$ and $\chi$ of the Poincaré representation of the principal polarization state of the light. It is well known that the relation between this representation and the Stokes parameter is the following 26 :

$$
\begin{aligned}
I & =S_{0}, \\
\mathscr{P} & =\left(S_{1}^{2}+S_{2}^{2}+S_{3}^{2}\right)^{1 / 2} / S_{0}, \\
\varphi & =\frac{1}{2} \tan ^{-1}\left(S_{2} / S_{1}\right), \\
\chi & =\frac{1}{2} \sin ^{-1}\left(S_{3} / I \mathscr{P}\right) .
\end{aligned}
$$

Based on the segmentation results obtained in Fig. 8, the estimated parameters of the objects and of their surroundings are listed in Table 1 . It can be seen that the average intensities of the objects and of the surrounding backgrounds are almost identical: There is no intensity contrast between the objects and the background. Furthermore, the principal polarization states in the objects and in the background are similar. Indeed, the observed surfaces lie in approximately the same plane, and it can be conjectured that the direction and ellipticity of the polarized part of the light is due to the Fresnel reflection, whose polarimetric characteristics are linked to the angle of incidence of the light on the surface and to the dielectric constant. In fact, it can be seen from Table 1 that the main contrast is due to the degree of polarization. The objects, which are pieces of transparent tape, depolarize the light less than does the coarser cardboard.

The proposed method is thus able to detect and segment objects based on their polarimetric properties. By construction, it takes as an input the last three components of the Stokes vector and performs
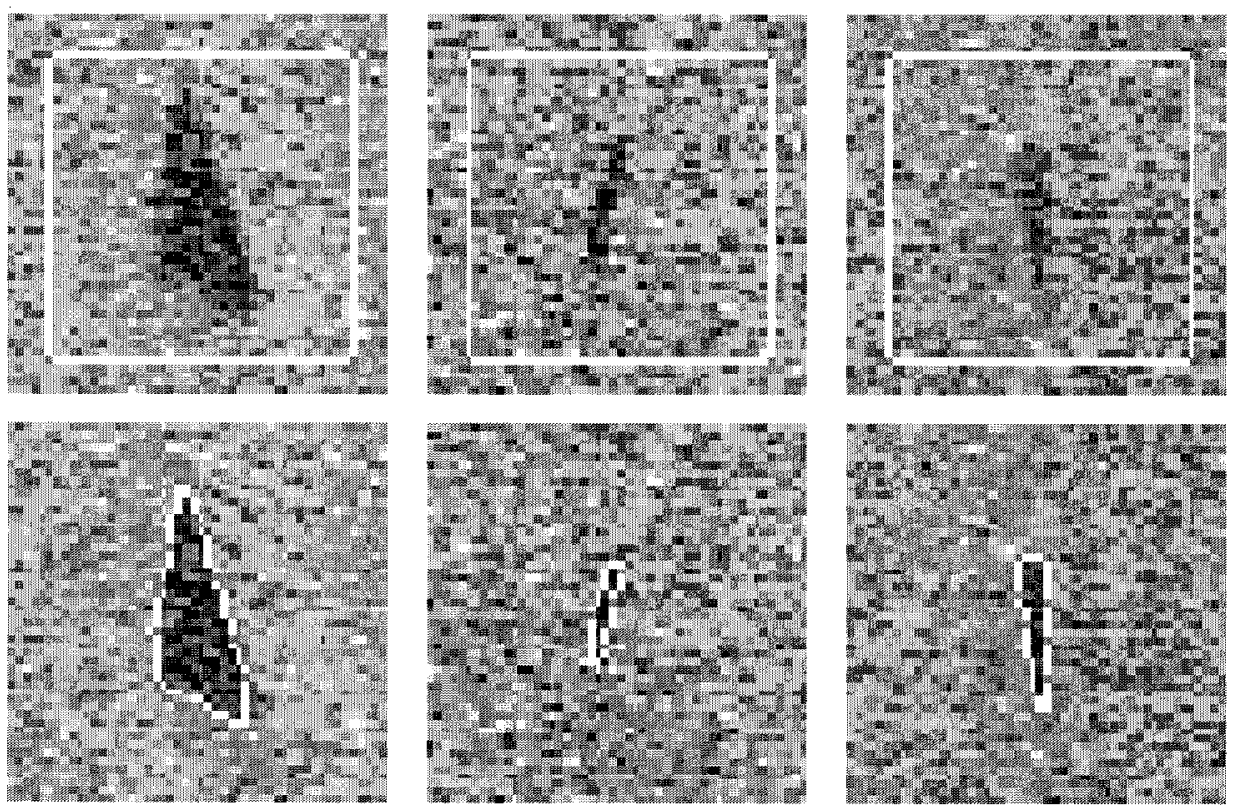

Fig. 8. Result of the segmentation of the three objects detected in the scene of Fig. 3. Top, initial snake; bottom, result of the segmentation. 
Table 1. Average Polarimetric Parameters of the Objects Segmented in Fig. $8^{a}$

\begin{tabular}{|c|c|c|c|c|c|c|}
\hline \multirow{2}{*}{$\begin{array}{c}\text { Polarimetric } \\
\text { Parameter }\end{array}$} & \multicolumn{2}{|c|}{ Object 1} & \multicolumn{2}{|c|}{ Object 2} & \multicolumn{2}{|c|}{ Object 3} \\
\hline & Object & Background & Object & Background & Object & Background \\
\hline$I$ & 183 & 181 & 185 & 182 & 184 & 182 \\
\hline $\mathscr{P}$ & 0.037 & 0.014 & 0.039 & 0.015 & 0.038 & 0.015 \\
\hline$\varphi\left({ }^{\circ}\right)$ & 73 & 82 & 74 & 81 & 73 & 81 \\
\hline$\chi\left(^{\circ}\right)$ & 2.5 & -2.9 & 2.35 & -1.9 & 2.7 & -2.1 \\
\hline
\end{tabular}

${ }^{a}$ The parameters are averaged over the regions determined by the snake segmentation algorithm. Polarimetric parameters $I$ (intensity), $\mathscr{P}$ (degree of polarization), and $\varphi$ and $\chi$ (polar angles) are derived from the average Stokes vectors estimated from each region.

detection based on this information. One does not need to know a priori in which Stokes parameter-or in which combination of Stokes parameters-a contrast exists. In the example given here, it was deduced a posteriori (that is, after segmentation) that the relevant information was the degree of polarization, but this information was not required for performing the segmentation: The same algorithms are also efficient if the contrast is in terms of polarization direction or ellipticity.

\section{Conclusions}

We have presented a Stokes imaging system based on a variable retardance scheme and liquid-crystal polarization modulators that acquires Stokes parameter images at a standard video rate. Because of the quality of the modulators, this system can detect faint variations of the polarimetric properties of the observed materials and thus reveal contrasts that do not appear in standard intensity images. To extract information efficiently from the vectorial Stokes parameter images we used statistics-based detectors and segmentation algorithms based on statistical active contours and the MDL principle. These algorithms were adapted to the processing of such noisy images, and we have shown some examples of their performance with real-world data.

The global system composed of the Stokes imaging system and of the processing algorithm can be used for such applications as default detection in industrial process control. Of course, it can be improved in many ways. In particular, calibration is a key point in such devices, ${ }^{8}$ and work to automate this operation is under way.

This research was performed in the framework of Multi-Laboratory Project Team 8, "Polarization imagery, from the components to the processing," of the department of Sciences et Technologies de l'Information et la Communication of the Centre $\mathrm{Na}$ tional de la Recherche Scientifique, whose support is gratefully acknowledged. The authors thank Pierre Ambs, Julien Charreyron, Philippe Réfrégier, and Jihad Zallat for fruitful discussions.

\section{References}

1. J. E. Solomon, "Polarization imaging," Appl. Opt. 20, 15371544 (1981).

2. W. G. Egan, W. R. Johnson, and V. S. Whitehead, "Terrestrial polarization imagery obtained from the Space Shuttle: char- acterization and interpretation," Appl. Opt. 30, 435-442 (1991).

3. L. B. Wolff, "Polarization camera for computer vision with a beam splitter," J. Opt. Soc. Am. A 11, 2935-2945 (1994).

4. J. S. Tyo, M. P. Rowe, E. N. Pugh, and N. Engheta, "Target detection in optical scattering media by polarization-difference imaging," Appl. Opt. 35, 1855-1870 (1996).

5. F. Goudail and Ph. Réfrégier, "Statistical algorithms for target detection in coherent active polarimetric images," J. Opt. Soc. Am. A 18, 3049-3060 (2001).

6. A. F. Sadjadi and C. S. L. Chun, "Automatic detection of small objects from their infrared state-of-polarization vectors," Opt. Lett. 28, 531-533 (2003).

7. L. B. Wolff and T. E. Boult, "Constraining object features using a polarization reflectance model,” IEEE Trans. Pattern Anal. Mach. Intell. 13, 635-657 (1991).

8. P. Terrier and V. DeVlaminck, "Robust and accurate estimate of the orientation of partially polarized light from a camera sensor," Appl. Opt. 40, 5233-5239 (2001).

9. T. Rogne, S. Stewart, and M. Metzler, "Infrared polarimetry: what, why, how and the way ahead," presented at the Third NATO IRIS Joint Symposium, Quebec, Canada (October 1998).

10. A. F. Sadjadi and C. S. L. Chun, "Passive polarimetric IR classification," IEEE Trans. Aerosp. Electron. Syst. 37, 740751 (2001).

11. K. Koshikawa, "A polarimetric approach to shape understanding of glossy objects," presented at the Sixth International Joint Conference on Artificial Intelligence, Tokyo, Japan (1979).

12. B. F. Jones and P. T. Fairney, "Recognition of shiny dielectric objects by analyzing the polarization of reflected light," Image Vision Comput. 7, 253-258 (1989).

13. L. B. Wolff, "Polarization vision: a new sensory approach to image understanding," Image Vision Comput. 15, 81-93 (1997).

14. L. B. Wolff, "Polarization-based material classification from specular reflection," IEEE Trans. Pattern Anal. Mach. Intell. 12, 1059-1071 (1990).

15. R. A. Chipman, "Polarimetry," in Handbook of Optics, M. Bass, ed. (McGraw-Hill, New York, 1995), pp. 22.1-22.33.

16. O. Ruch and $\mathrm{Ph}$. Réfrégier, "Minimal-complexity segmentation with a polygonal snake adapted to different optical noise models," Opt. Lett. 41, 977-979 (2001).

17. J. S. Tyo, "Design of optimal polarimeters: maximization of SNR and minimization of systematic errors," Appl. Opt. 41, 619-630 (2002).

18. C. Brosseau, Fundamentals of Polarized Light-A Statistical Approach (Wiley, New York, 1998).

19. J. J. Drewes, R. A. Chipman, and M. H. Smith, "Characterizing polarization controllers with Mueller matrix polarimetry," in Active and Passive Optical Components for WDM Communication, A. K. Dutta, A. A. S. Awwal, N. K. Dutta, and K. Okamoto, eds., Proc. SPIE 4532, 462-466 (2001). 
20. J. Zallat, Y. Takakura, and M.-Ph. Stoll, "Active imaging polarimetry," presented at the Physics in Signal and Image Processing Conference 2001, Marseille, France (2001).

21. H. V. Poor, "Elements of hypothesis testing," in An Introduction to Signal Detection and Estimation (Springer-Verlag, New York, 1994), pp. 5-39.

22. V. Pagé, F. Goudail, and Ph. Réfrégier, "Improved robustness of target location in nonhomogeneous backgrounds by use of the maximum likelihood ratio test location algorithm," Opt. Lett. 24, 1383-1385 (1999).

23. O. Germain and Ph. Réfrégier, "On the bias of the likelihood ratio edge detector for SAR images," IEEE Trans. Geosci. Remote Sens. 38, 1455-1458 (2000).

24. O. Germain and Ph. Réfrégier, "Optimal snake-based segmentation of a random luminance target on a spatially disjoint background," Opt. Lett. 21, 1845-1847 (1996).

25. C. Chesnaud, Ph. Réfrégier, and V. Boulet, "Statistical region snake-based segmentation adapted to different physical noise models," IEEE Trans. Pattern Anal. Mach. Intell. 21, 11451157 (1999).

26. S. Huard, "Polarized optical wave," in Polarization of Light (Masson, Paris, 1997), pp. 1-35. 